\title{
MODULACIÓN DE LOS NIVELES DE LIPOPROTEÍNAS DE ALTA DENSIDAD Y LAS CITOQUINAS IL-1ß E IL-6 EN PACIENTES CON DENGUE
}

\author{
Estefanía Barrientos-Arenas ${ }^{1,2, a}$, Vanessa Henao-García ${ }^{1,2, a}$, Diana Marcela Giraldo ${ }^{2, b}$, Margarita María Cardona ${ }^{3, c}$, \\ Silvio Urcuqui-Inchima ${ }^{2, d}$, Jhon Carlos Castaño ${ }^{3, e}, J^{2}$ Juan C. Hernández ${ }^{1,2, f}$
}

\begin{abstract}
RESUMEN
Objetivos. Evaluar los niveles de lipoproteínas de alta densidad (HDL) y citoquinas proinflamatorias en pacientes con dengue clasificados según la gravedad del cuadro clínico. Materiales y métodos. Se realizó un estudio descriptivo, incluyendo 70 individuos con dengue, clasificados como: sin signos de alarma (DSSA), con signos de alarma (DCSA) y dengue grave (DG); 15 donantes sanos fueron incluidos como controles (CS). Los niveles séricos de IL-1 $1 \beta$ e IL-6 fueron cuantificados por ELISA, y los de HDL en suero por ensayo colorimétrico; además se determinó el recuento de plaquetas y el porcentaje de hematocrito. Resultados. Los niveles de IL-1 $\beta$ e IL-6 se encontraron aumentados en los pacientes con DENV respecto a los controles sanos. Dicho aumento fue más evidente en los pacientes con dengue, de acuerdo con la gravedad del cuadro clínico. Los niveles de HDL se hallaron disminuidos en los pacientes con dengue comparados con los controles sanos. Los análisis de correlación mostraron asociaciones estadísticamente significativas entre HDL y el número de plaquetas; así como entre el porcentaje de hematocrito y los niveles de IL-1 $\beta$. Conclusiones. El aumento de IL-1 $\beta$ e IL-6 y la disminución de HDL en los estadios clínicos más graves sugieren que ambos factores están implicados en el desarrollo de la patogénesis y severidad. Las correlaciones permiten observar una posible asociación entre HDL y el número de plaquetas que permite dar una aproximación al posible papel de las HDL en la patogénesis del dengue, pero aún es necesario realizar estudios adicionales que permitan elucidar su importancia en el curso de la infección por DENV.
\end{abstract}

Palabras claves: Lipoproteínas de alta densidad; Dengue; Interleucina-1beta; Interleucina-6; Inflamación (fuente: DeCS BIREME)

\section{MODULATION OF HIGH-DENSITY LIPOPROTEIN AND CYTOKINE IL-1 $\beta$ AND IL-6 LEVELS IN PATIENTS WITH DENGUE}

\begin{abstract}
Objectives. To assess High-Density Lipoprotein (HDL) and pro-inflammatory cytokine levels in patients with dengue classified according to the severity of the clinical presentation. Materials and Methods. A descriptive study was carried out with 70 individuals with dengue, classified as no alarm signs (DSSA), with signs of alarm (DCSA), and severe dengue (DG); 15 healthy donors were included as controls (CS). The serum levels of IL- $1 \beta$ and IL-6 were quantified through ELISA, and the HDL levels through serum by a colorimetric test. In addition, the platelet count and the hematocrit percentage were determined. Results. Levels of IL-1 $\beta$ and IL- 6 were found to be increased in patients with DENV vs. the healthy controls. Said increase was more evident in patients with dengue and in line with the severity of the clinical presentation. The HDL levels were found to be decreased in patients with dengue versus healthy controls. The correlation analysis showed statistically significant associations between HDL and the platelet number, as well as between the hematocrit percentage and the levels of IL-1 $1 \beta$. Conclusions. The increased IL-1 $\beta$ and IL-6 and the decreased HDL levels in the most severe clinical stages suggest that both factors are involved in the development of the pathogenesis and severity. The correlations allow the observation of a potential association between $\mathrm{HDL}$ and platelet count, which allows us to provide an approach to the possible role of HDL in the pathogenesis of dengue, but it is still necessary to perform additional studies that will allow to state their importance in the course of the DENV infection.
\end{abstract}

Keywords: High-density lipoproteins; Dengue; Interleukin-1beta; Interleukin-6; Inflammation (source: MeSH NLM)

\footnotetext{
Infettare, Facultad de Medicina, Universidad Cooperativa de Colombia. Medellín, Colombia.

Grupo Inmunovirología, Facultad de Medicina, Universidad de Antioquia. Medellín, Colombia.

Grupo Inmunología Molecular (GYMOL), Facultad Ciencias de la Salud, Universidad del Quindío. Quindío, Colombia.

a Estudiante de Microbiología; ${ }^{\mathrm{b}}$ bióloga, MSc; ${ }^{\mathrm{c}}$ bacteriologa. MSc; ${ }^{\mathrm{d}}$ biólogo, PhD; ${ }^{\mathrm{e}}$ médico, Doc.Sci; ${ }^{\mathrm{f}}$ microbiólogo y bioanalista, Doc.Sci.

Recibido: 11/08/2017 Aprobado: 07/02/2018 En línea: 23/03/2018
}

Citar como: Barrientos-Arenas E, Henao-García V, Giraldo DM, Cardona MM, Urcuqui-Inchima S, Castaño JC, et al. Modulación de los niveles de lipoproteínas de alta densidad y las citoquinas IL-1 $\beta$ e IL-6 en pacientes con dengue. Rev Peru Med Exp Salud Publica. 2018;35(1):15-24. doi: 10.17843/rpmesp.2018.351.3568. 


\section{INTRODUCCIÓN}

El virus dengue (DENV) pertenece al género Flavivirus, de la familia Flaviviridae; hasta la fecha se han descrito cuatro serotipos (DENV1 a DENV4). El virus es transmitido por la picadura de mosquitos (hembras infectadas), principalmente Aedes albopictus y Aedes aegypti, los cuales coexisten en el continente americano, se ha descrito que este último cobra mayor importancia por su predominio en áreas urbanas ${ }^{(1)}$. El dengue ha sido considerado un problema prioritario para la salud pública del mundo, debido al número de casos reportados al año y la población ubicada en zonas geográficas de riesgo (principalmente áreas tropicales y subtropicales). Para la semana epidemiológica 41 de 2017 , la Organización Panamericana de la Salud reportó 22476 casos de dengue (6104 confirmados por laboratorio) en Colombia ${ }^{(2)}$. Los pacientes con dengue son clasificado en tres cuadros clínicos de acuerdo la Organización Mundial de la Salud (OMS) i) dengue sin signos de alarma (DSSA), donde se presenta un malestar general inespecífico; ii) dengue con signos de alarma (DCSA), que incluyen dolor abdominal, hepatomegalia, aumento del hematocrito $y$ trombocitopenia; y iii) dengue grave (DG), esta última presentación amenaza incluso la vida del paciente debido a la posibilidad de desarrollar hemorragias masivas y falla multiorgánica ${ }^{(3)}$.

En el contexto de la infección por el DENV, la respuesta inflamatoria implica la participación de células de la inmunidad innata como monocitos y células dendríticas, las cuales en respuesta a la infección son activadas, modulando la expresión de receptores innatos, que llevan a la producción de citoquinas proinflamatorias como IL-1 $1 \beta$ e IL-6, entre otras ${ }^{(4,5)}$. Estas citoquinas son importantes para contener la diseminación viral durante los primeros días de la infección. Sin embargo, la alteración en el balance de la producción de citoquinas proinflamatorias es uno de los marcadores de la patogénesis en la infección con DENV (6). La falta de regulación de este proceso, así como su prolongación, puede afectar la homeostasis de los tejidos, lo cual incrementa la permeabilidad vascular, induce perdida de fluidos y mayores complicaciones clínicas ${ }^{(7)}$. La ausencia de tratamientos específicos para los pacientes con dengue hace necesaria la búsqueda de nuevas estrategias terapéuticas que modulen el proceso infeccioso. En los últimos años se ha sugerido búsqueda de moléculas y sustancias endógenas que contribuyan a un mejor pronóstico.

Entre estas moléculas, las HDL (lipoproteínas de alta densidad) han tomado importancia, estas son las encargadas del transporte reverso del colesterol, es decir el movimiento de los excesos de colesterol desde los tejidos periféricos hasta el hígado para su posterior eliminación. Este proceso implica la transferencia de colesterol desde las células, en los diferentes tejidos, a

\section{MENSAJES CLAVE}

Motivación para realizar el estudio. El dengue se caracteriza por la respuesta inflamatoria, que desencadena las complicaciones clínicas; y aún no existen terapias que mejoren su desenlace clínico. Las lipoproteínas de alta densidad (HDL), pueden regular la inflamación, lo que podría favorecer en el curso de la enfermedad.

Principales hallazgos. En comparación con individuos sanos, los pacientes con dengue presentan menores niveles de HDL y mayores concentraciones de las citoquinas proinflamatorias IL-1 $\beta$ e IL-6, especialmente aquellos con los cuadros clínicos más graves.

Implicancias. La modulación de las HDL podría representar una nueva alternativa terapéutica en los pacientes con dengue.

las HDL a través de las proteínas ABCA-1 (ATP-binding cassette transporter $\mathrm{G} 1$ ), la esterificación de colesterol mediada por la enzima LCAT (lecitin colesterol acetil transferase), la transferencia de ésteres de colesterol a otras lipoproteínas y la eliminación hepática a través de receptores scavenger (SR-B) ${ }^{(8)}$ (Anexo 1. Disponible en: https://goo.gl/2MyDnj)

Por otro lado, se ha descrito que las HDL tienen propiedades antiinflamatorias, disminuyendo la expresión de citoquinas proinflamatorias como TNF- $\alpha$, IL-1 $\beta$ e IL- 6 en monocitos y macrófagos ${ }^{(9)}$. Además, pueden modular la expresión de moléculas de adhesión en las células endoteliales en respuesta al estímulo de algunos receptores de la inmunidad innata, como los receptores tipo Toll (TLR) y tipo NOD (NLR), estando posiblemente relacionados con el secuestro del colesterol intracelular afectando las balsas lipídicas y la señalización intracelular ${ }^{(8)}$.

Las HDL han adquirido importancia en el contexto de la patogénesis del dengue, ya que se han reportado cambios en el perfil lipídico durante el curso de la infección por DENV ${ }^{(10)}$, los cuales dependen de la severidad de la enfermedad ${ }^{(11)}$. Se ha observado que los niveles de HDL y LDL (lipoproteínas de baja densidad) se encuentran disminuidas en pacientes con dengue grave comparado con individuos sanos y en pacientes con dengue sin signos de alarma ${ }^{(12,13)}$. De acuerdo con estos hallazgos, se ha postulado que estas lipoproteínas podrían utilizarse como predictores de la progresión clínica del dengue ${ }^{(11)}$. A pesar de estos hallazgos, aún se desconoce el papel que juegan las HDL en la patogénesis de la infección por DENV, y es poco lo que se conoce sobre la interacción del virus con estas lipoproteínas. Se ha demostrado que las HDL favorecen la entrada del DENV a la célula, mediada por uno de sus principales componentes, la ApoA-I, (apolipoproteína $\mathrm{Al})$, la cual se asocia con las partículas virales promoviendo la infección al facilitar la entrada del virus a la célula diana ${ }^{(14)}$.

Basados en estos antecedentes y teniendo en cuenta que, tanto las HDL como las citoquinas proinflamatorias se alteran durante el curso de la infección por el DENV, el 
objetivo del presente estudio fue evaluar el comportamiento de estos componentes en los diferentes estadios clínicos del dengue y explorar si existe una asociación de los niveles séricos de HDL y las citoquinas pro-inflamatorias IL-1 $\beta$ e IL-6, incluso con parámetros clínicos importantes en el seguimiento de los pacientes con dengue, como el recuento de plaquetas y el porcentaje de hematocrito.

\section{METODOLOGÍA}

\section{DISEÑO Y POBLACIÓN DE ESTUDIO}

Se realizó un estudio descriptivo transversal, con un enfoque cuantitativo y retrospectivo, utilizando muestras previamente recolectadas en un hospital público de la ciudad de Armenia (Colombia), con diagnóstico de infección con DENV, en diferentes estadios clínicos. El diagnóstico de laboratorio fue dado por la detección del antígeno viral NS1 o la detección de lgM e lgG específica en suero, determinado a través de la prueba rápida SD BIOLINE DENV Duo (NS1 Ag and lgM/lgG Alere, Australia).

En total se incluyeron 70 muestras de suero (almacenadas en alícuotas a $-80^{\circ} \mathrm{C}$ ) de pacientes con diagnóstico de dengue clasificados de acuerdo a los lineamientos de la OMS, como se describe a continuación: dengue sin signos de alarma $(n=22)$, dengue con signos de alarma $(n=37)$ y dengue grave $(n=11)$ según los parámetros clínicos y de laboratorio reportados en las historias clínicas de los pacientes incluyendo: manifestaciones hemorrágicas, trombocitopenia, efusión pleural y cambios en el hematocrito, entre otros.
Además, se incluyeron 15 muestras de suero obtenidas de donantes clínicamente sanos, con prueba rápida para diagnóstico de dengue negativo, y sin antecedentes previos de enfermedad febril en los últimos dos meses, y sin antecedentes de enfermedades metabólicas (dislipidemias, diabetes e hipertensión arterial) (Tabla 1).

\section{CRITERIOS DE INCLUSIÓN Y EXCLUSIÓN}

En el estudio se contemplaron como criterios de inclusión que las muestras fueran provenientes de individuos de ambos sexos, afiliados a alguno de los regímenes en salud y que presentaran alguna de las condiciones clínicas del estudio. No se incluyó en el estudio pacientes con diagnóstico de dislipidemias, embarazo, cáncer o no cumplieran con las condiciones preanalíticas.

\section{VARIABLES}

\section{Cuantificación de HDL}

Los niveles séricos de HDL fueron cuantificados con un ensayo colorimétrico, usando el estuche comercial HDLprecipitante (Biosystems S.A. Barcelona), siguiendo las instrucciones del fabricante. Brevemente, las muestras fueron mezcladas con el reactivo precipitante de HDL, y luego centrifugadas por 10 minutos a 4000 rpm. Posteriormente, al sobrenadante se le adicionó el reactivo de colesterol, y se incubó a temperatura ambiente durante 10 minutos. Los resultados se leyeron a una longitud de onda de $490 \mathrm{~nm}$ y se reportaron en $\mathrm{mg} / \mathrm{dL}$.

Tabla 1. Características clínicas y demográficas de los participantes del estudio

\begin{tabular}{|c|c|c|c|c|}
\hline Características & $\begin{array}{c}\text { CS } \\
(n=15)\end{array}$ & $\begin{array}{l}\text { DSSA } \\
(n=22)\end{array}$ & $\begin{array}{l}\text { DCSA } \\
(n=37)\end{array}$ & $\begin{array}{c}\text { DG } \\
(n=11)\end{array}$ \\
\hline Masculino/Femenino & $2 / 13$ & $15 / 7$ & $20 / 17$ & $8 / 3$ \\
\hline $\begin{array}{l}\text { Edad (años) } \\
\text { mediana (RIC) }\end{array}$ & $39(30-44)$ & $28(19-52)$ & $27(12-52)$ & $55(21-64)$ \\
\hline $\begin{array}{l}\text { Días de evolución } \\
\text { mediana (RIC) }\end{array}$ & - & $6(5-8)$ & $6(7-6)$ & $7(6-7)$ \\
\hline $\begin{array}{l}\text { Plaquetas (plaquetas } / \mathrm{mm}^{3} \text { ) } \\
\text { mediana (RIC) }\end{array}$ & - & $45200(11000-224000)$ & $94000(7000-278000)$ & $20500(9000-63000)$ \\
\hline $\begin{array}{l}\text { Hemoconcentración* (\%) } \\
\text { mediana (RIC) }\end{array}$ & - & $3,5(1,7-8,2)$ & $4,7(2,7-8,3)$ & $5,3(2,6-11,2)$ \\
\hline Hepatomegalia o Esplenomegalia (\%) & - & 0,0 & 5,4 & 27,3 \\
\hline Petequias (\%) & - & 13,6 & 32,4 & 27,3 \\
\hline Hemorragias espontáneas ${ }^{\dagger}(\%)$ & - & 18,2 & 54,1 & 9,1 \\
\hline Edema pulmonar (\%) & - & 0,0 & 5,4 & 27,3 \\
\hline
\end{tabular}

DSSA: denguesin signos de alarma, DCSA: dengue con signos de alarma, DG: dengue grave, RIC: Rango intercuartílico, CS: Controles sanos

* Hemoconcentración: Porcentaje de hemoconcentración, al comparar la muestra inicial (día 0) y la muestra control (día 3).

† Hemorragias espontáneas definido como presencia de epixtasis, hemoptisis, o equimosis. 


\section{Cuantificación de IL-1 $\beta$ e IL-6}

Los niveles séricos de IL-1 $\beta$ e IL-6 se cuantificaron por el método Enzyme-Linked ImmunoSorbent Assay (ELISA) usando un estuche comercial específico para cada citoquina (BD Biosciences, Franklin Lakes, NJ), y siguiendo las instrucciones previamente reportadas ${ }^{(15)}$. Los resultados se leyeron a una longitud de onda de $450 \mathrm{~nm}$ y se reportaron en $\mathrm{pg} / \mathrm{ml}$.

Asimismo, se incluyó la cuantificación del hematocrito (\%) y el recuento de plaquetas $\left(\mathrm{mm}^{3}\right)$.

\section{ANÁLISIS ESTADÍSTICO}

Para el análisis estadístico se utilizó el programa GraphPad Prism ${ }^{\circledR} 5.0$ (San Diego, CA, USA). La normalidad y homocedasticidad fueron evaluadas usando las pruebas de Shapiro-Wilky Levene, respectivamente. Para la descripción de las variables cuantitativas se calcularon medidas de resumen (mediana y rangos intercuartílicos) y para las variables cualitativas frecuencias absolutas y relativas. Las pruebas $\mathrm{U}$ de Mann-Whitney y Kruskal-Wallis fueron usadas para las comparaciones entre los grupos. En los casos de significancia estadística en la prueba de Kruskal-Wallis, se realizó además la prueba de Dunn (Post hoc). Para los análisis de correlación se utilizaron las pruebas de Pearson - Spearman (para datos paramétricos o no paramétricos, respectivamente). En todos los análisis se consideró un nivel de significación estadística $(p)$ menor a 0,05.

\section{ASPECTOS ÉTICOS}

El manejo de la información personal de cada paciente fue de total discreción y se usó exclusivamente para dar cumplimiento a los objetivos previamente planteados. De acuerdo con los lineamientos éticos para investigación, todos los participantes firmaron un consentimiento informado y en el caso de los menores de edad fueron sus padres los que brindaron el consentimiento para participar en el estudio. Además, todos los procedimientos fueron realizados de acuerdo con los principios de la declaración de Helsinki. El estudio fue aprobado por el comité de ética de la Universidad Cooperativa de Colombia.

\section{RESULTADOS}

Inicialmente se evaluaron los niveles séricos de IL-1 $\beta$ e IL-6 por ELISA en el grupo control y en el total de pacientes con dengue (sin tener en cuenta la presentación clínica). Como se observa en la Figura 1 , los pacientes con dengue presentaron mayores niveles de IL-1 $\beta$, diferencias que fueron significativas comparadas con el control $(p=0,032)$, con una diferencia total de la mediana en una magnitud de $1,58 \mathrm{pg} / \mathrm{ml}$. El mismo comportamiento fue observado para IL-6, la cual fue significativamente mayor en los pacientes con dengue, comparado con los controles $(p<0,0001$, tamaño del efecto: $13,38 \mathrm{pg} / \mathrm{ml}$ ).

El total de pacientes con dengue fueron clasificados según las manifestaciones clínicas, los niveles séricos de IL-1 $\beta$ e IL-6 fueron determinados por ELISA para cada grupo de individuos. Para ambas citoquinas se observó un aumento estadísticamente significativo en suero $(p=0,003$ y $p<0,0001$, respectivamente) según la severidad clínica, el cual se mantiene entre el grupo de pacientes con DG comparado con el control, para ambas citoquinas (Figura 2).

En el presente estudio, se observó que los niveles séricos de HDL estaban significativamente disminuidos en los pacientes con dengue, comparado con los controles sanos a.

$\mathrm{IL}-1 \beta$

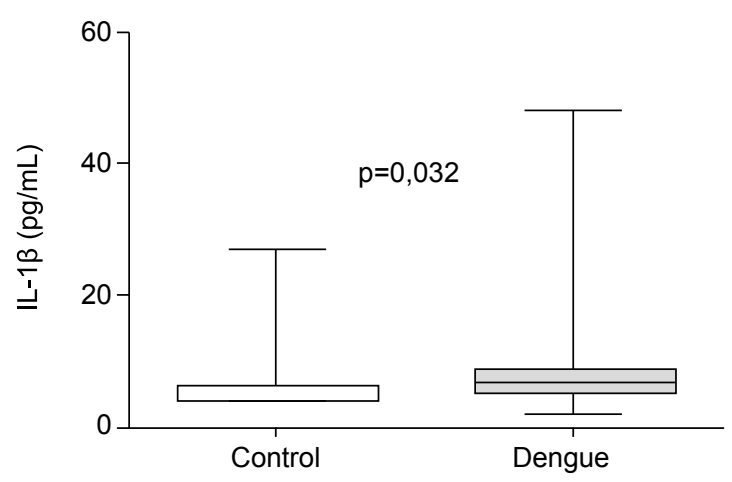

b.

IL-6

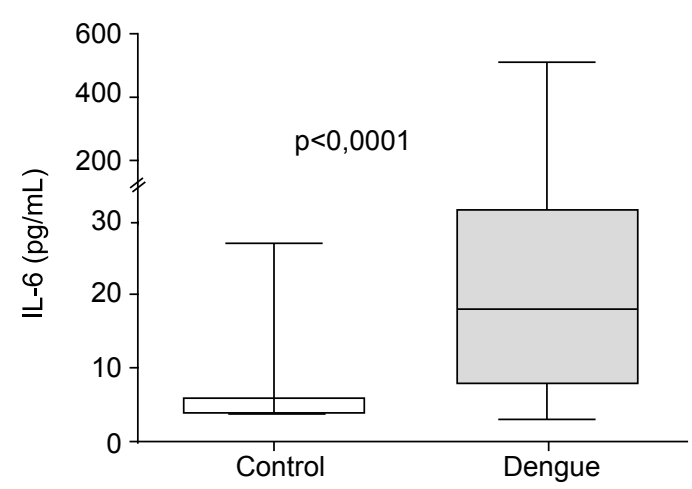

Figura 1. Niveles séricos de IL-1 $\beta$ e IL-6 (pg/mL) en los participantes del estudio. Los niveles séricos de IL-1 1 (a) e IL-6 (b) fueron cuantificados por ELISA en controles $(n=15)$ y en pacientes con dengue $(n=70)$, los resultados fueron reportados en $\mathrm{pg} / \mathrm{mL}$. Se hicieron comparaciones usando la prueba de $\mathrm{U}$ de Mann-Whitney. 
a.

IL-1 $\beta$

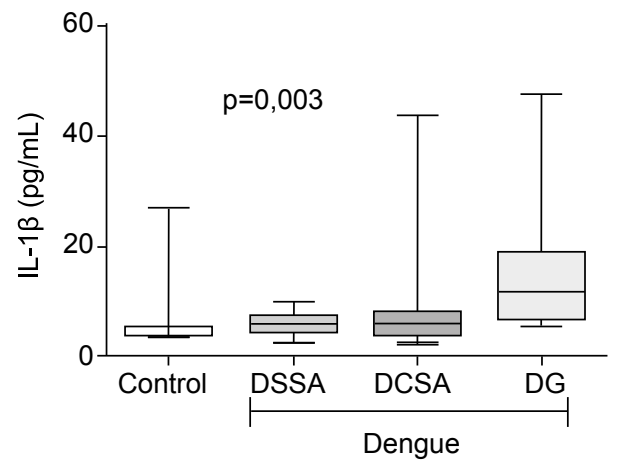

b.

IL-6

Figura 2. Niveles séricos de IL-1 $\beta$ e IL-6 $(\mathrm{pg} / \mathrm{mL})$ en controles sanos y pacientes con dengue según su clasificación clínica. Los niveles de IL-1ß (a) e IL-6 (b) fueron comparados usando la prueba de KruskalWallis y el post hoc Dunn.

Controles ( $n=15)$, DSSA: dengue sin signos de alarma $(n=22)$, DCSA: dengue con signos de alarma $(n=37)$ y DG: dengue grave $(n=11)$

(Figura 3a) ( $p=0,0032$, tamaño del efecto: $13,12 \mathrm{mg} / \mathrm{dL})$. De igual manera, cuando se realizó el análisis según la gravedad de la enfermedad, se observó una disminución significativa en los pacientes con DSSA y DCSA, comparados con los controles sanos (Figura $3 b)(p=0,006)$. Sin embargo, no se observaron diferencias estadísticamente significativas en los niveles de las HDL entre los pacientes con DG y los controles sanos. No se observó diferencias significativas en los niveles de HDL entre hombres y mujeres (datos no mostrados), sugiriendo que el sexo no es un factor determinante en los resultados reportados. No obstante, se debe considerar la diferencia en la proporción de hombres y mujeres en cada uno de los grupos de estudio (Tabla 1).
Con los resultados obtenidos sobre la producción de IL-1 $\beta$ e IL-6 y con los niveles de HDL, se procedió a determinar si existía una correlación entre esos valores y otros parámetros clínicos presentes en los pacientes con dengue. Se realizaron análisis de correlación entre HDL e IL-1 $\beta$ (Figura 4a) y HDLe IL-6 (Figura 4b) pero no se encontró significancia estadística. Adicionalmente se exploró la correlación de los niveles de HDL con dos parámetros clínicos importantes en el curso de la infección por DENV como el porcentaje de hematocrito y el recuento de plaquetas. Aunque el porcentaje de hematocrito no se correlacionó con las HDL (Figura 4c), el recuento de plaquetas y las HDL (Figura 4d) presentaron una correlación positiva baja, estadísticamente a.

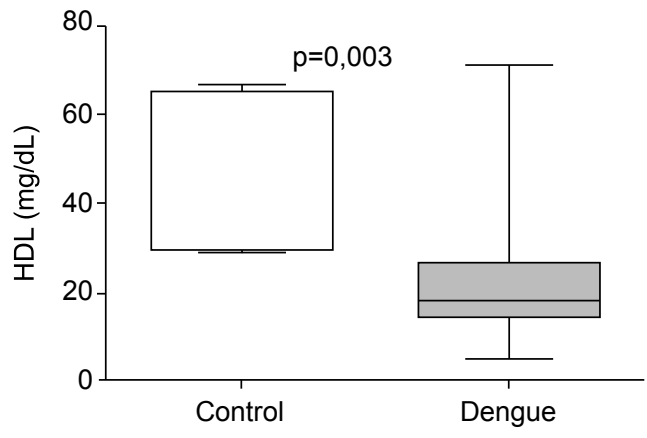

b.

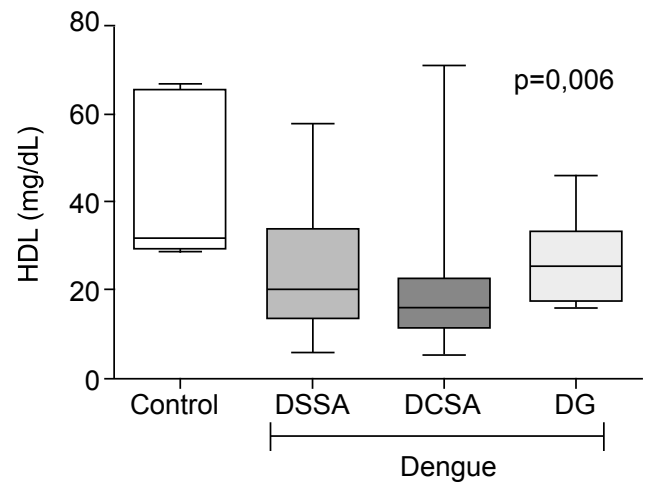

Figura 3. Niveles séricos de HDL ( $\mathrm{mg} / \mathrm{dL}$ ) en los participantes del estudio. Los niveles de HDL fueron comparados entre el grupo control $(n=15)$ y el total pacientes con dengue $(n=70)(a)$; o cada uno de los grupos de pacientes según su clasificación clínica (b). DSSA: dengue sin signos de alarma $(n=22)$, DCSA: dengue con signos de alarma $(n=37)$ y DG: dengue grave $(n=11)$. Se hicieron comparaciones usando las pruebas Mann-Whitney $U$ o Kruskal-Wallis (con el post hoc Dunn), respectivamente. 

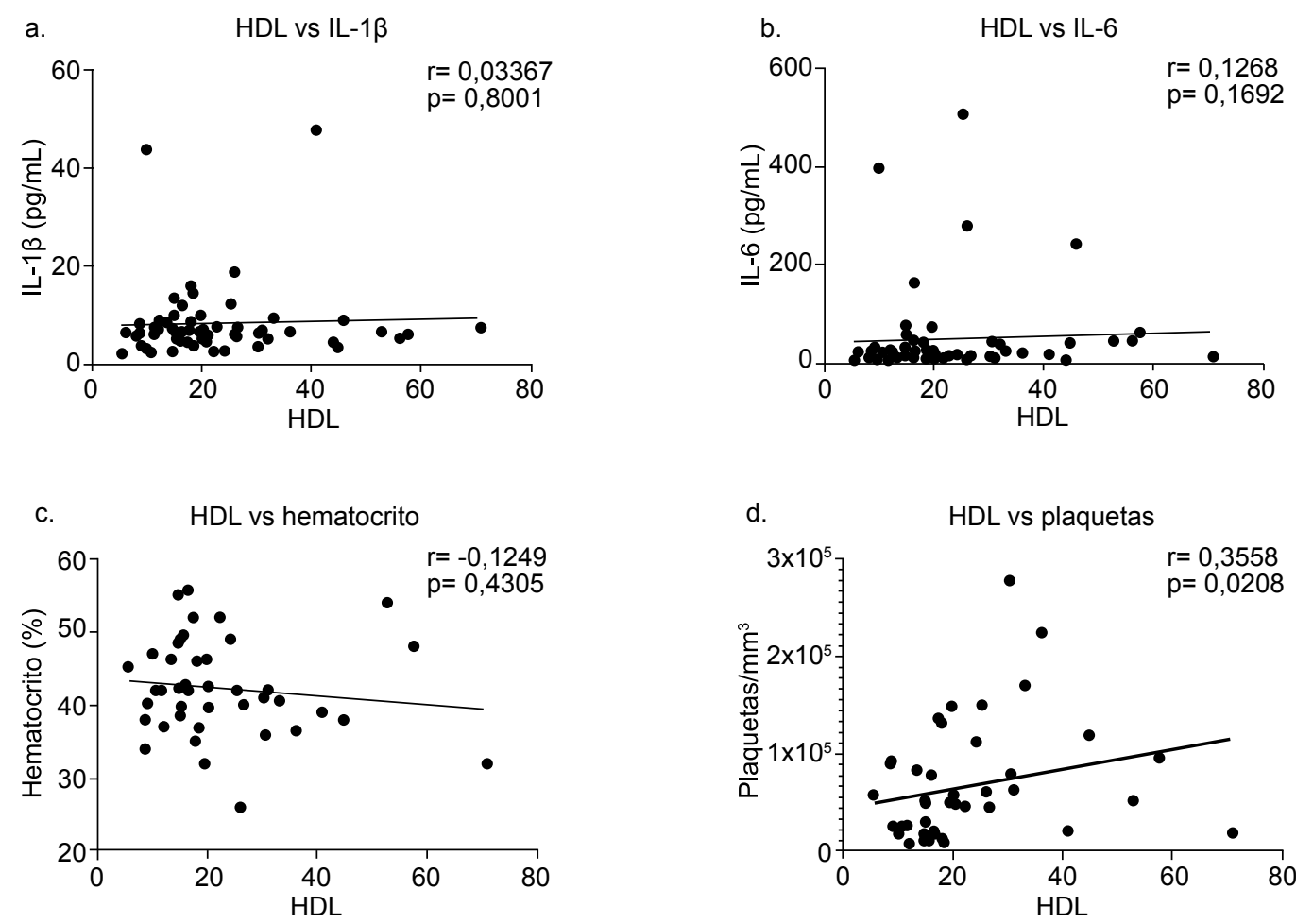

Figura 4. Análisis de correlación de los niveles de HDL con las variables IL-1 $\beta$, IL-6, hematocrito y recuento de plaquetas. Los niveles de HDL en suero fueron correlacionados con la concentración sérica de IL-1 $\beta$, IL-6, el porcentaje de hematocrito y el recuento de plaquetas en el total de pacientes con dengue. Se realizó una prueba de normalidad a los datos analizados, para los datos que presentaban una distribución no normal se aplicó una prueba de correlación de Spearman; por otro lado, para los datos que presentaron una distribución normal se realizó la prueba de correlación de Pearson.

significativa. Los análisis de correlación no evidenciaron resultados significativos cuando se analizaron de acuerdo con la gravedad del cuadro clínico (DSSA, DCSA y DG) (datos no mostrados).

Se evaluó el porcentaje de hematocrito en cada grupo clínico, pero no fueron observadas diferencias significativas entre los grupos (Figura 5a). Igualmente, se realizaron análisis de correlación entre el porcentaje de hematocrito e IL-1 $\beta$ (Figura 5b), IL-6 (Figura 5c) y el número de plaquetas (Figura $5 \mathrm{~d}$ ), aunque se observaron tendencias, sólo se encontró correlación negativa baja, estadísticamente significativa con IL-1 $\beta$.

\section{DISCUSIÓN}

En la dinámica de la respuesta inmune frente a la infección por DENV, la respuesta inflamatoria es la principal característica de la patogénesis viral. La producción exacerbada de citoquinas proinflamatorias, como IL-12, IL-17, IL-6, TNF- $\alpha$ e IL-1 $\beta$ (16,17), contribuyen de manera diferencial en el curso de la infección por DENV, por un lado induciendo la diferenciación y proliferación de células plasmáticas y direccionando la respuesta inmune adaptativa y por otro, favoreciendo el desarrollo de la patogénesis del dengue, induciendo la activación del endotelio vascular e incrementando la permeabilidad vascular, fenómenos claves que contribuyen al desarrollo de una enfermedad más grave ${ }^{(7)}$.

Acorde a la literatura previa ${ }^{(18)}$, en este estudio se evidenció un aumento significativo en las concentraciones séricas de las citoquinas proinflamatorias IL-6 e IL- $1 \beta$ en los pacientes con dengue cuando se comparó con el grupo control. Este resultado era el esperado ya que el desarrollo de la patogénesis en la infección por DENV se caracteriza por una alta inducción de citoquinas proinflamatorias. Estas citoquinas participan en todo el proceso inflamatorio observado en los pacientes con dengue y en algunos casos, este proceso no es controlado por el organismo, afectando el normal funcionamiento de las células endoteliales, lo que incrementa la permeabilidad endotelial y promueve perdida de plasma ${ }^{(19)}$. Estos eventos finalmente son los que en gran medida determinan las diferentes presentaciones clínicas del dengue: DSSA, DCSA y DG ${ }^{(20)}$. En esos pacientes se observó un aumento en la producción de IL-1 $\beta$ e IL-6, especialmente en los que tenían DCSA y DG, tal como fue reportado previamente ${ }^{(18)}$, lo que indica que un ambiente proinflamatorio exagerado contribuye a la gravedad de la patogénesis del dengue. 
a.
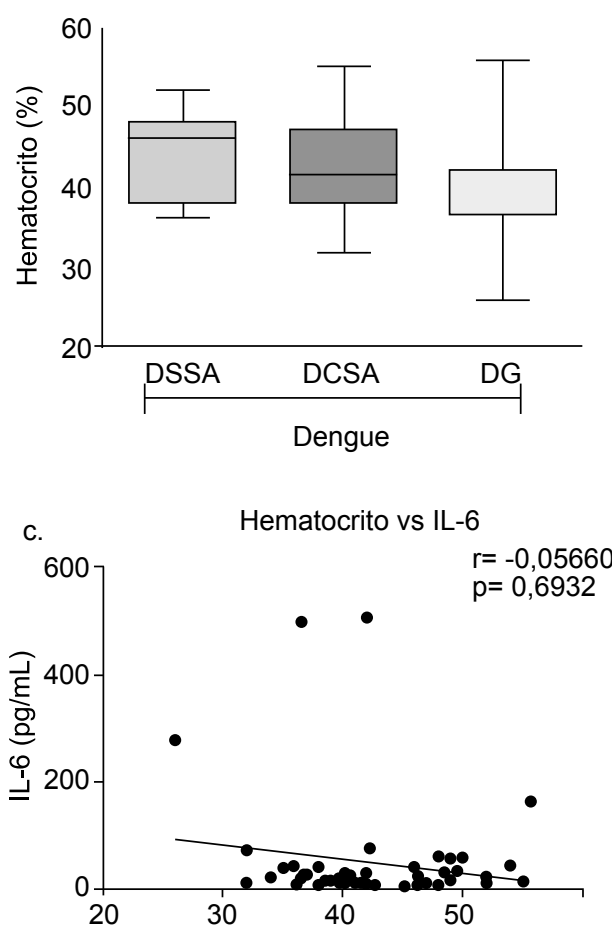

b. Hematocrito vs IL-1 $1 \beta$
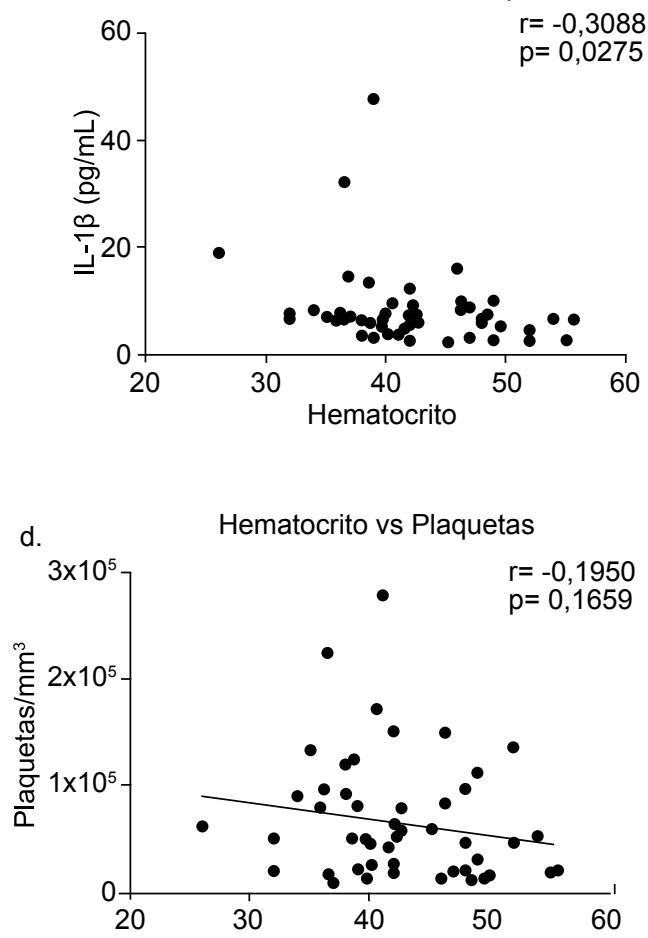

Figura 5. Análisis de correlación del porcentaje de hematocrito con las variables IL-1 $\beta$, IL-6 y recuento de plaquetas. El porcentaje de hematocrito fue correlacionado con la concentración sérica de IL-1 $\beta$, IL-6 y el recuento de plaquetas en el total de pacientes con dengue. Se realizó una prueba de normalidad a los datos analizados, para los datos que presentaban una distribución no normal se aplicó una prueba de correlación de Spearman; para los datos que presentaron una distribución normal se realizó la prueba de correlación de Pearson.

El papel biológico de estas citoquinas y las implicancias en la severidad de la patogénesis por dengue ha sido discutido previamente; IL-1 $\beta$ por ejemplo, es el principal mediador de la activación de las células endoteliales inducida por plaquetas y la IL-6 contribuye potenciando la cascada de coagulación (18). El incremento de las citoquinas proinflamatorias se ha mencionado en varios estudios ${ }^{(6)}$, donde incluso se ha descrito como una "tormenta de citoquinas", o sobreproducción de diversos mediadores inflamatorios, presentes en la forma clínica más grave del dengue ${ }^{(21)}$. La presencia de estas citoquinas proinflamatorias ocasiona además la infiltración de células mononucleares en órganos como el hígado, pulmón y riñón, demostrando el compromiso de estos órganos, lo que puede inducir falla del mismo y ocasionar la muerte ${ }^{(22)}$. Dichos eventos inmunopatológicos asociados con una fuerte respuesta inflamatoria han sido descritos previamente, incluso para otros agentes virales como el virus del oeste de $\mathrm{Nilo}^{(23)}$, que al igual que DENV, pertenece a la familia Flaviviridae.

Las HDL son ampliamente reconocidas, no sólo por el transporte reverso del colesterol, sino porque cumplen otras funciones como antioxidantes, antiinflamatorias, e inhibidores de procesos como la apoptosis o la trombosis, por lo que se les ha atribuido una función ateroprotectora ${ }^{(24)}$. Incluso algunos autores han evidenciado la necesidad del aumento farmacológico de las HDL ${ }^{(25)}$, como una estrategia para contrarrestar el desarrollo de la placa ateromatosa y por consiguiente, a disminuir la respuesta inflamatoria producida durante la enfermedad ${ }^{25)}$. En cuanto a las implicancias de las HDL en las infecciones virales, previamente se reportó que la función antioxidante de las HDL de los pacientes infectados por $\mathrm{VIH}-1$ se encontraba alterada, en comparación con los individuos sanos, probablemente debido a menores niveles de ApoA-I (principal componente proteico de las HDL) ${ }^{(26)}$. Esos estudios mostraron como las HDL eran reguladas en diferentes condiciones patológicas.

En el presente estudio se demostró que las HDL estaban disminuidas significativamente en pacientes con dengue comparados con el grupo control, y siendo mayor la diferencia en los pacientes con DG. Estudios previos realizados, igualmente en pacientes con dengue también observaron el mismo comportamiento en los niveles de las HDL, con una disminución mayor en los individuos con manifestaciones clínicas más graves ${ }^{(11-13)}$. Si bien, los diferentes estudios reportan que durante la infección 
por DENV, las HDL son moduladas negativamente, el papel de estas en el curso de la patogénesis permanece desconocido. Aunque el sexo podría considerarse como una variable de confusión en estos análisis, en el presente estudio no se observaron diferencias significativas entre hombre y mujeres, en cuanto a los niveles de HDL ${ }^{(27)}$.

Con el propósito de profundizar en el conocimiento del papel de las HDL durante la inmunopatogénesis del dengue, con los resultados obtenidos se realizaron análisis de correlación entre los niveles de HDL y las citoquinas proinflamatorias, el porcentaje de hematocrito y el recuento de plaquetas. Se encontró una correlación positiva baja entre los niveles de HDL y el recuento de plaquetas, lo que evidencia que a menor número de plaquetas se observa menores niveles de HDL, panorama característico del desarrollo de un cuadro clínico más grave por dengue. Esto toma importancia ya que se ha descrito que las HDL previenen el daño y la muerte de células endoteliales, inhibe activación de plaquetas y la cascada de coagulación ${ }^{(28)}$, sugiriendo que los bajos niveles de HDL y el bajo número de plaquetas conllevan a una presentación más grave de la infección por DENV. Esto es muy interesante si se tiene en cuenta que el daño endotelial es el principal factor desencadenante de los eventos hemorrágicos observados en las formas graves de la enfermedad. En conjunto, estos resultados muestran que la disminución de HDL se correlaciona con la gravedad de la enfermedad por dengue, sugiriendo que las HDL podrían retrasar el desarrollo clínico más severo de la infección por DENV. Sin embargo, es necesario realizar estudios longitudinales que permitan conocer la dinámica del metabolismo de las HDL, en especial en el contexto de la infección por DENV. Además, es importante considerar las características propias de la población estudiada (incluyendo datos sociodemográficos e inmunogenéticos, entre otros), ya que estas variables podrían influenciar los resultados, al realizar estudios poblacionales en diferentes localizaciones geográficas.

El hematocrito es otro parámetro modulado en el curso de la infección por DENV, incluso este parámetro es utilizado clínicamente para enmarcar la gravedad de la enfermedad por dengue, aunque este puede verse afectado por variables como el sexo y la edad, entre otras ${ }^{(29)}$. Por lo tanto, se evaluó si el porcentaje de hematocrito se correlacionaba con las citoquinas proinflamatorias de interés o con el número de plaquetas. Se encontró una correlación negativa baja entre el porcentaje de hematocrito y los niveles de IL-1 $\beta$. Esto puede ser contradictorio ya que en las formas más severas de dengue, lo esperado es que haya un mayor porcentaje de hematocrito y mayores niveles de IL-1 ${ }^{(30)}$; sin embargo, en la población estudiada los niveles de hematocrito disminuían a mayor severidad. Este fenómeno podría explicarse por la necesidad de hospitalización en algunos pacientes con los cuadros clínicos más graves, que además requirieron el uso de líquidos intravenosos durante su estancia.

Tanto el recuento de plaquetas como el porcentaje de hematocrito han sido previamente reportados como parámetros predictivos de los cuadros clínicos graves en los pacientes con dengue ${ }^{(30)}$. Sin embargo, en el presente estudio no se encontró correlación significativa entre estas variables. Estos resultados pueden explicarse por la presencia de edema pulmonar en algunos pacientes, donde la pérdida de plasma podría reflejar el aumento de los niveles de hematocrito. Además, la trombocitopenia refleja la dimensión de los diferentes mecanismos inflamatorios durante la fase aguda y su relación también se asocia con la extravasación del plasma, pero esta correlación no fue observada en los pacientes.

Una de las limitaciones del estudio, fue el tamaño de muestra que incluyó 85 individuos, y aunque los análisis estadísticos permiten plantear algunas hipótesis, aumentar el tamaño de muestra e incluir seguimiento a los pacientes con mediciones de laboratorio en diferentes tiempos, podría contribuir al mejor entendimiento de la relación entre las HDL y la respuesta inflamatoria en estos pacientes. Otro aspecto que podría aportar información relevante es la cuantificación de otras citoquinas proinflamatorias, como TNF- $\alpha$, la cual es importante en la inmunopatogénesis de la infección por el virus dengue; sin embargo, las citoquinas evaluadas IL-1 $\beta$ e IL-6 representan ampliamente el perfil inflamatorio reportado durante el dengue.

En conclusión, en el presente estudio se muestra que factores como las HDL y las citoquinas proinflamatorias evaluadas son moduladas principalmente en los pacientes que presentan las manifestaciones graves de la enfermedad y que, junto con otros parámetros como trombocitopenia y la hemoconcentración, son determinantes en el curso clínico de la infección por el DENV. No obstante, aún es necesario realizar más estudios que demuestren el papel de las HDL dentro de la patogénesis del dengue, que permitan conocer el mecanismo por el cual estas son moduladas y si en alguna medida influyen en el cambio de otros parámetros bien descritos en el curso de la infección por dengue.

Contribuciones de autoría: EBA, VHG y JCH son los responsables del diseño de proyecto e interpretación de los datos. EBA y VHG realizaron los análisis de laboratorio. EBA, VHG, DMG, JCH y SUI participaron en el análisis de resultados y preparación del manuscrito. MMC y JCC participaron en la recolección de las muestras y evaluación final del manuscrito. Todos los autores revisaron y aprobaron la versión final del manuscrito.

Conflictos de interés: Los autores declaran no tener conflictos de interés.

Fuente de financiación: Este trabajo fue financiado por Colciencias, Proyecto 141565741029. 


\section{REFERENCIAS BIBLIOGRÁFICAS}

1. Rey JR, Lounibos P. Ecología de Aedes aegypti y Aedes albopictus en América y transmisión de enfermedades. Biomedica. 2015;35(2):177-85. doi: 10.1590/S012041572015000200005 .

2. PHOWHO. Reported Cases of Dengue Fever in th Americas, by Country or Territory, Epidemiological information as of October 27. Geneva: PHO, WHO; 2017. Disponible en: http://www.paho. org/hq/index.php?option $=$ com_docman\&task=doc_view\&gid $=42728 \&$ Ite $\mathrm{mid}=270\langle=\mathrm{en} 2017$

3. Organizacióa Panamericana de la Salud, Organización Mundial de la Salud. Dengue: Guías para el diagnóstico, tratamiento, prevención y control [Internet]. La Paz: OPS, OMS; 2010. Disponible en: http://apps.who.int/iris/bitst ream/10665/44504/1/9789995479213 spa.pdf?ua $=1$

4. Martinez J, HernandezJC, Urcuqui-Inchima S. Papel de las células dendríticas en la infección por el virus dengue: blancos de replicación y respuesta inmune. Rev Chilena Infectol. 2017;34(3):249-256. doi: 10.4067/S0716-10182017000300007.

5. Torres S, Hernández JC, Giraldo D, Arboleda M, Rojas M, Smit JM, et al. Differential expression of Toll-like receptors in dendritic cells of patients with dengue during early and late acute phases of the disease. PLoS Negl Trop Dis. 2013;7(2):e2060. doi: 10.1371/journal. pntd.0002060.

6. Villar LÁ, Gélvez RM, Rodríguez JA, Salgado D, Parra B, Osorio L, et al. Biomarcadores pronósticos de gravedad del dengue. Biomedica. 2013;33 Suppl 1:108-16.

7. Appanna R, Wang SM, Ponnampalavanar SA, Lum LC, Sekaran SD. Cytokine factors present in dengue patient sera induces alterations of junctional proteins in human endothelial cells. Am J Trop Med Hyg. 2012;87(5):936-42. doi: 10.4269/ajtmh.2012.11-0606.

8. Marín-Palma D, Taborda NA, UrcuquiInchima S, Hernandez JC. Inflamación y respuesta inmune innata: participación de las lipoproteínas de alta densidad. Iatreia. 2017;30(4):424-36. doi: 10.17533/udea. iatreia.v30n4a06.
9. Säemann MD, Poglitsch M, Kopecky C, Haidinger M, Hörl WH, Weichhart $\mathrm{T}$. The versatility of HDL: a crucial anti-inflammatory regulator. Eur J Clin Invest. 2010;40(12):1131-43. doi: 10.1111/j.1365-2362.2010.02361.x.

10. Suvarna JC, Rane PP. Serum lipid profile: a predictor of clinical outcome in dengue infection. Trop Med Int Health. 2009;14(5):576-85. doi: 10.1111/j.13653156.2009.02261.x.

11. van Gorp EC, Suharti C, Mairuhu AT, Dolmans WM, van Der Ven J, Demacker $\mathrm{PN}$, et al. Changes in the plasma lipid profile as a potential predictor of clinical outcome in dengue hemorrhagic fever. Clin Infect Dis. 2002;34(8):1150-3.

12. Biswas HH, Gordon A, Nuñez A, Perez MA, Balmaseda A, Harris E. Lower LowDensity Lipoprotein Cholesterol Levels Are Associated with Severe Dengue Outcome. PLoS Negl Trop Dis. 2015 Sep 3;9(9):e0003904. doi: 10.1371/journal. pntd.0003904.

13. Durán A, Carrero R, Parra B, González A, Delgado L, Mosquera J, et al. Association of lipid profile alterations with severe forms of dengue in humans. Arch Virol. 2015;160(7):1687-92. doi: 10.1007/ s00705-015-2433-z.

14. Li Y, Kakinami C, Li Q, Yang B, Li H. Human apolipoprotein A-I is associated with dengue virus and enhances virus infection through SR-BI. PLoS One. 2013;8(7):e70390. doi: 10.1371/journal. pone. 0070390 .

15. Arboleda-Alzate JF, Rodenhuis-Zybert IA, Hernández JC, Smit JM, Urcuqui-Inchima S. Human macrophages differentiated in the presence of vitamin D3 restrict dengue virus infection and innate responses by downregulating mannose receptor expression. PLoS Negl Trop Dis. 2017;11(10):e0005904. doi: 10.1371/ journal.pntd.0005904.

16. Becquart P, Wauquier N, Nkoghe D, Ndjoyi-Mbiguino A, Padilla C, Souris M, et al. Acute dengue virus 2 infection in Gabonese patients is associated with an early innate immune response, including strong interferon alpha production. BMC Infect Dis. 2010;10:356. doi: 10.1186/1471-2334-10-356.
17. Callaway JB, Smith SA, Widman DG, McKinnon KP, Scholle F, Sempowski GD, et al. Source and Purity of DengueViral Preparations Impact Requirement for Enhancing Antibody to Induce Elevated IL-1 $\beta$ Secretion: A Primary Human Monocyte Model. PLoS One. 2015;10(8):e0136708. doi: 10.1371/ journal.pone. 0136708 .

18. Martina BE, Koraka P, Osterhaus AD. Dengue virus pathogenesis: an integrated view. Clin Microbiol Rev. 2009;22(4):56481. doi: 10.1128/CMR.00035-09.

19. Stephenson JR. Understanding dengue pathogenesis: implications for vaccine design. Bull World Health Organ. 2005;83(4):308-14.

20. Organización Panamericana de la Salud. Dengue: Guías para la atención de enfermos en la región de las Américas [Internet]. 2da Ed. Washington, DC: OPS; 2016. Disponible en: http:// iris.paho.org/xmlui/bitstream/handle/123456789/28232/9789275318904 esp.pdf ?sequence $=1$ \&isAllowed $=y$

21. Basu A, Chaturvedi UC. Vascular endothelium: the battlefield of dengue viruses. FEMS Immunol Med Microbiol. 2008;53(3):287-99. doi: 10.1111/j.1574695X.2008.00420.x

22. Póvoa TF, Oliveira ER, Basílio-de-Oliveira CA, Nuovo GJ, Chagas VL, Salomão NG, et al. Peripheral Organs of Dengue Fatal Cases Present Strong Pro-Inflammatory Response with Participation of IFN-Gamma-, TNF-Alpha- and RANTES-Producing Cells. PLoS One. 2016;11(12):e0168973. doi: 10.1371/ journal.pone.0168973.

23. Diosa-Toro M, Hernandez JC. Immunopathogenesis of West Nile Virus Infection. Curare. 2014;1:17-23. doi: 10.16925/cu.v1i2.925.

24. Calderón JU. Perspectivas del HDL colesterol, como objetivo en el llamado riesgo cardiovascular residual. Rev peru cardiol. 2010;26(1):1-17.

25. Badimón JJ, Ibáñez B. Incremento de las HDL como arma terapéutica en aterotrombosis. Rev Esp Cardiol. 2010;63(03):323-33.

26. Kelesidis T, Oda MN, Borja MS, Yee Y, Ng KF, Huynh D, et al. Predictors 
of impaired HDL function in HIV1 infected compared to uninfected individuals. J Acquir Immune Defic Syndr. 2017;75(3):354-363. doi: 10.1097/ QAI.0000000000001383.

27. Pascot A, Lemieux I, Bergeron J, Tremblay A, Nadeau A, Prud'homme D, et al. HDL particle size: a marker of the gender difference in the metabolic risk profile. Atherosclerosis. 2002;160(2):399-406.
28. deGoma EM, deGoma RL, Rader DJ. Beyond high-density lipoprotein cholesterol levels evaluating high-density lipoprotein function as influenced by novel therapeutic approaches. J Am Coll Cardiol. 2008;51(23):2199-211. doi: 10.1016/j.jacc.2008.03.016.

29. Díaz-Quijano FA, Martinez-Vega RA, Villar-Centeno LA. Asociación entre la magnitud del viraje del hematocrito y otros indicadores de severidad en Dengue. Colomb Med. 2009; 40(4):408-14.

30. Yacoub S, Wills B. Predicting outcome from dengue. BMC Med. 2014;12:147. doi: 10.1186/s12916-014-0147-9.

Correspondencia: Juan Carlos Hernández Dirección: Infettare, Universidad Cooperativa de Colombia, Calle 50 N 40-74. Medellin, Colombia Teléfono: 57 (4) 4446065 extensión 4245

Correo electrónico:juankhernandez@gmail.com 


\section{ANEXO 1. METABOLISMO DE LAS HDL}
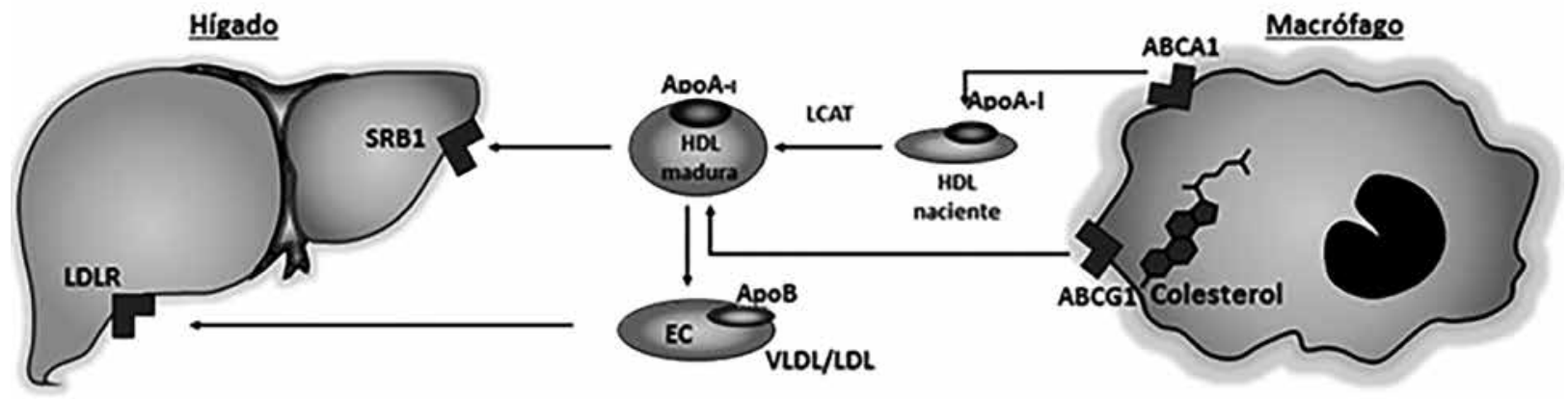

La apolipoproteína AI (apoA-I) es la principal proteína estructural de las HDL, la cual capta colesterol libre de los tejidos periféricos a través de la proteína transportadora dependiente de ATP 1 (ABCA1), dando lugar a la HDL naciente. Éstas HDL nacientes maduran a las formas esféricas mediante la obtención de colesterol libre y fosfolípidos a través de la proteína ABCG1 (ATP binding cassette transporter G1), la esterificación del colesterol por la enzima Lecitin colesterol acil transferasa (LCAT) y el intercambio de ésteres de colesterol por triglicéridos con otras lipoproteínas. El proceso finaliza por medio de dos vías: i) Las HDL maduras ingresan al hígado a través del receptor scavenger clase B tipo 1 (SR-B1) o ii) transfiere el colesterol a las apoproteínas B-100 (ApoB), transformándose en lipoproteínas de muy baja densidad/ lipoproteínas de baja densidad (VLDL/LDL), que a su vez se unen a los receptores de LDL (LDL-R) presentes en el hígado 